\title{
Author Correction: RPAP3 provides a flexible scaffold for coupling HSP9O to the human R2TP co-chaperone complex
}

Fabrizio Martino1, Mohinder Pal ${ }^{2}$, Hugo Muñoz-Hernández ${ }^{1,3}$, Carlos F. Rodríguez ${ }^{1,3}$, Rafael Núñez-Ramírez David Gil-Carton ${ }^{4}$, Gianluca Degliesposti ${ }^{5}$, J. Mark Skehel ${ }^{5}$, S. Mark Roe ${ }^{2}$, Chrisostomos Prodromou ${ }^{2}$, Laurence H. Pearl (1) ${ }^{2} \&$ Oscar Llorca (i) 1,3

Correction to: Nature Communications https://doi.org/10.1038/s41467-018-03942-1; published online 16 April 2018

In the originally published version of this article, the affiliation details for Hugo Muñoz-Hernández, Carlos F. Rodríguez and Oscar Llorca incorrectly omitted 'Centro de Investigaciones Biológicas (CIB), Spanish National Research Council (CSIC), Ramiro de Maeztu 9, 28040 Madrid, Spain'. This has now been corrected in both the PDF and HTML versions of the Article.

Published online: 31 July 2018

(i) Open Access This article is licensed under a Creative Commons Attribution 4.0 International License, which permits use, sharing, adaptation, distribution and reproduction in any medium or format, as long as you give appropriate credit to the original author(s) and the source, provide a link to the Creative Commons license,

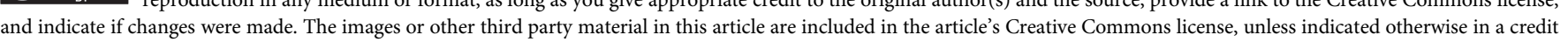

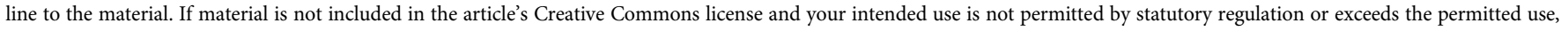
you will need to obtain permission directly from the copyright holder. To view a copy of this license, visit http://creativecommons.org/licenses/by/4.0/.

(C) The Author(s) 2018

\footnotetext{
${ }^{1}$ Centro de Investigaciones Biológicas (CIB), Spanish National Research Council (CSIC), Ramiro de Maeztu 9, 28040 Madrid, Spain. ${ }^{2}$ Genome Damage and Stability Centre, School of Life Sciences, University of Sussex, Falmer, BN1 9RQ Brighton, UK. ${ }^{3}$ Spanish National Cancer Research Centre (CNIO), Melchor Fernández Almagro 3, 28029 Madrid, Spain. ${ }^{4}$ Structural Biology Unit, CIC bioGUNE, Bizkaia Technology Park, 48160 Derio, Spain. ${ }^{5}$ MRC Laboratory of Molecular Biology, Cambridge Biomedical Campus, Francis Crick Avenue, CB2 OQH Cambridge, UK. These authors contributed equally: Fabrizio Martino, Mohinder Pal, Hugo Muñoz-Hernández and Carlos F. Rodríguez. Correspondence and requests for materials should be addressed to L.H.P. (email: Laurence.Pearl@sussex.ac.uk) or to O.L. (email: ollorca@cnio.es)
} 